\title{
Effect of sulfur aggregates on mechanical resistance and durability for SFRHPC with the addition of slag
}

\section{Effet des granulats sulfurés sur les résistances mécanique et la durabilité des BHPF avec ajouts de laitier}

\author{
Boutiba Aldjia ${ }^{1}$, Chaid Rabah ${ }^{2}$, Moulez Laurent ${ }^{3}$ et Jauberthie Raoul ${ }^{3}$ \\ ${ }^{1}$ Dpt. Architecture, Faculté du Génie de la Construction, Université de Tizi Ouzou \\ ${ }^{2}$ Unité de recherche : Matériaux - Procédés et Environnement, Université de Boumerdès \\ ${ }^{3}$ Laboratoire de Génie Civil et Génie Mécanique (LGCGM), INSA-Rennes, Université Européenne de Bretagne, France
}

\begin{abstract}
The transformation of sulfides present in the aggregate to sulfates causes internal sulfate attack (ISA) by formation of secondary ettringite in the hardened concrete. This pathological ettringite crystallizes, the generated pressure is greater than the tensile strength of the concrete. It generates internal swelling and causes disorders that can severely damage the structures. Favors to their structural and economic performance, high performance concrete steel fiber (SFRHPC) are increasingly used in construction. Increase productivity and reduce construction time on site. They provide substantial weight savings, therefore it is possible to build with less formwork, less concrete to set up and fewer reinforcement than ordinary concrete. The purpose of this study is to determine the effect of sulfur present in the hornfels crushed aggregates, on the mechanical strength, durability, and the microstructure of SFRHPC with the addition of blast furnace slag. In ordinary concrete, the pyrite aggregates cause cracking by expansion when they are in wet land. In high performance concretes this phenomenon is greatly reduced or absent. This is the result of a very low porosity, reduced flow of moisture that cannot propagate to aggregate. And sulfide is stable and cannot be transformed into aggressive sulfate.
\end{abstract}

Résumé : La transformation en sulfates des sulfures présents dans les granulats, provoquent la réaction sulfatique interne (RSI) par formation d'éttringite secondaire (tardive) dans le béton durci. Cette ettringite pathologique cristallise, la pression engendrée est supérieure à la résistance à la traction du béton. Elle génère des gonflements internes et cause des désordres susceptibles d'endommager sévèrement les ouvrages. Grâce à leurs performances structurales et économiques, les bétons de hautes performances de fibres métalliques (BHPF), sont de plus en plus utilisés dans la construction. Ils permettent d'augmenter la productivité et de réduire les délais de réalisation sur chantiers. Ils procurent des gains de masse conséquents, il est donc possible de construire avec moins de coffrage, moins de béton à mettre en place et moins d'armatures qu'avec les bétons ordinaires. Le but de cette étude, est de définir l'effet du soufre présent dans les granulats de concassage de roches cornéennes, sur les résistances mécaniques, la durabilité, ainsi que la microstructure des BHPF avec ajout de laitier de haut fourneau. Dans les bétons ordinaires, les granulats pyriteux provoquent de la fissuration par expansion lorsqu'ils sont en milieu humide. Dans les bétons à hautes performances ce phénomène est fortement réduit ou inexistant. Ceci est la conséquence d'une porosité très réduite, une circulation de l'humidité réduite qui ne peut se propager jusqu'au granulat. Ainsi le sulfure est stable et ne peut se transformer en sulfate agressif. 


\section{Introduction}

Les roches utilisées pour la fabrication des granulats peuvent contenir des sulfures dont la nature et l'abondance varient en fonction de leurs conditions de genèse. Le rôle nocif des sulfures de fer dans les granulats, utilisés pour la confection des bétons a été mis en évidence par Berard et al, Oberholster et al. [1, 2]. La transformation en sulfates des sulfures présents dans les granulats, provoquent, une réaction sulfatique interne (RSI) par formation d'éttringite secondaire (tardive) dans le béton durci [3]. Cette ettringite pathologique cristallise, la pression de cristallisation engendrée est supérieure à la résistance en traction du béton. Elle génère des gonflements internes et cause des désordres susceptibles d'endommager sévèrement les ouvrages $[3,4]$.

La RSI touche essentiellement les parties d'ouvrage en contact avec l'eau ou exposé à un taux d'humidité élevé. Les travaux de Heinz et Ludwig [5] et de Graf [6] ont montré qu'aucune expansion n'est observée en dessous de $90 \%$ d'humidité relative. Cependant, une teneur de $100 \%$ d'humidité relative permet le développement d'expansion.

Grâce à leurs performances structurales et économiques, les bétons de hautes performances de fibres métalliques sont de plus en plus utilisés dans la construction. Ils permettent d'augmenter la productivité et de réduire les délais de réalisation sur les chantiers. Ils procurent des gains de masse conséquents, il est possible de construire avec moins de coffrage, moins de béton à mettre en place et moins d'armatures qu'avec les bétons ordinaires. Cela a été montré pour les BHP et les BTHP (gains de 25 à 40\%) [7,8].

La confection d'un BHP nécessite un dosage en ciment moyen de $450 \mathrm{~kg} / \mathrm{m}^{3}$, la substitution d'une quantité de ciment par du laitier dans la composition de ce type de béton permet de limiter les émissions de gaz à effet de serre, de valoriser les déchets et d'économiser du ciment. Aussi plusieurs travaux [9, 10,11] ont montré que l'introduction des additions minérales telles que les laitiers de haut fourneau dans les bétons, en substitution au ciment, réduit voire annule l'expansion provoquée par la RSI.

Le travail présenté a été réalisée au laboratoire de génie civil et génie mécanique (LGCGM) de l'INSA de Rennes. Il a pour objet d'étudier l'effet du soufre présent dans les granulats de concassage de roches cornéennes sur les résistances mécaniques, la durabilité, ainsi que la microstructure des BHPF avec ajout de laitier de haut fourneau.

\section{Matériaux et méthodes}

Les matériaux utilisés pour la confection des bétons d'étude sont :

- Un ciment de type Portland sans ajouts minéraux, CEM I 52,5 PM ES CP2 de l'usine Lafarge du Teil (France). Les caractéristiques chimique et minéralogique sont reportées dans le tableau 1 .
Les propriétés physiques du ciment utilisé sont les suivantes :

Début de prise...........................2h 55mn

Retrait à 28jours........................560 $\mu \mathrm{m} / \mathrm{m}$

Masse volumique absolue.............. 3,16 g/cm

Stabilité chimique.........................0,5 mm

Surface spécifique.................... $3590 \mathrm{~cm}^{2} / \mathrm{g}$

- Le laitier utilisé est un sous-produit de la fabrication de la fonte, de l'usine d'El-Hadjar Annaba (Algérie). C'est un granulé amorphe, broyé jusqu'à une surface spécifique supérieure à celle du ciment, dont la composition chimique est reportée sur le tableau 2.

Les propriétés physiques du laitier sont les suivantes:

Masse volumique absolue .............. 2,99 g/ $\mathrm{cm}^{3}$

Surface spécifique ..................... $8500 \mathrm{~cm}^{2} / \mathrm{g}$

Tableau 1. Composition chimique et minéralogique du ciment

\begin{tabular}{|c|c|c|c|c|c|c|c|c|c|c|c|}
\hline 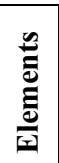 & $\begin{array}{l}0 \\
\tilde{J}\end{array}$ & $\stackrel{0}{N}_{i=1}^{N}$ & 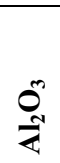 & ర్ల & $\sum_{\sum}^{\infty}$ & $\begin{array}{l}0 \\
\underline{2}\end{array}$ & 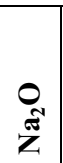 & in & $\bar{\simeq}$ & $\stackrel{\Sigma}{a}$ & $\begin{array}{l}0 \\
\text { లే }\end{array}$ \\
\hline$\partial^{\circ}$ & $\begin{array}{l}8 \\
: \\
:\end{array}$ & $\begin{array}{l}\stackrel{q}{+} \\
\text { i }\end{array}$ & $\begin{array}{l}\stackrel{0}{i} \\
\text { in }\end{array}$ & $\hat{n}$ & $\stackrel{n}{o}$ & $\begin{array}{l}n \\
0 \\
0\end{array}$ & $\begin{array}{l}\circ \\
0 \\
0\end{array}$ & $\stackrel{2}{i}$ & $\begin{array}{l}\text { กิ } \\
\text { o. }\end{array}$ & $\stackrel{\text { nे }}{=}$ & $\begin{array}{l}0 \\
\text { n. } \\
0\end{array}$ \\
\hline & & & & & $S$ & $6 \%$ & & & $\mathbf{A}=$ & & \\
\hline
\end{tabular}

RI: résidus insolubles, PAF: pertes au feu

- Un sable siliceux de concassage $(0 / 5)$, trois fraction de gravier $(4 / 6,3 ; 6,3 / 10 ; 10 / 14)$. Ces graviers sont issus du concassage de roches cornéennes contenant jusqu'à $0,1 \%$ de soufre, Bien que le pourcentage en soufre total soit faible $(0,1 \%)$, le souffre se trouve dans les microfissures de la roche et après le concassage ce soufre se répartit à la surface des granulats.

- Pour les bétons fibrés, on a utilisé un mélange de fibres courtes à raison de $40 \mathrm{~kg}$ par mètre cube de béton et des fibres à crochets (fibres encollées Dramix RC-80/50BN) à raison de $30 \mathrm{~kg}$ par mètre cube de béton.

Tableau 2. Composition chimique du laitier

\begin{tabular}{|c|c|c|c|c|c|c|c|c|c|c|}
\hline 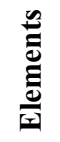 & ల్ల & $\stackrel{0}{N}$ & 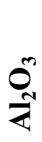 & 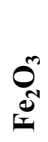 & ?.o & 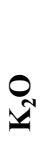 & $\begin{array}{l}0 \\
\tilde{z} \\
z\end{array}$ & $\stackrel{\wp}{\infty}$ & $\bar{\simeq}$ & $\frac{\sqrt{2}}{2}$ \\
\hline$d^{\circ}$ & $\stackrel{\hat{N}}{\hat{a}}$ & $\frac{\mathfrak{b}}{\vec{f}}$ & $\stackrel{2}{2}$ & $\underset{-}{F}$ & $\begin{array}{l}\mathfrak{g} \\
i\end{array}$ & $\underset{0}{\mathbb{Z}}$ & $\stackrel{0}{0}$ & $\frac{n}{0}$ & $\frac{1}{0}$ & $\exists$ \\
\hline
\end{tabular}

RI: résidus insolubles, PAF: pertes au feu

\subsection{Formulation des bétons et méthodes d'essais}

La formulation des bétons est réalisée à l'aide de la méthode de Dreux-Gorisse. Deux bétons ont été formulés : un béton de hautes performances (BHP) et un béton ordinaire (BO) comme matériaux de contrôle. Les compositions de base retenues pour ces deux bétons sont 
détaillées dans le tableau 3. Pour les bétons avec ajout, le laitier est substitué à raison de $15 \%$ de la masse du ciment.

Les éprouvettes des différents bétons, après démoulage, sont conservées jusqu'aux échéances d'essais dans une salle humide à la température de $20^{\circ} \mathrm{C}$ et une humidité relative supérieure à $95 \%$. Le tableau 4 synthétise la nomenclature des différents bétons avec substitution du ciment par du laitier et introduction de fibres.

Tableau 3 : Composition des bétons d'étude

\begin{tabular}{|c|c|c|c|}
\hline \multicolumn{2}{|r|}{ Bétons } & $\begin{array}{c}\text { Béton } \\
\text { ordinaire }\end{array}$ & $\begin{array}{l}\text { Béton de haute } \\
\text { performance }\end{array}$ \\
\hline \multirow{7}{*}{ 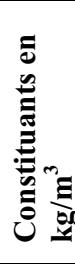 } & Ciment & 350 & 500 \\
\hline & Eau & 214 & 179 \\
\hline & Sable 0/5 & 763,89 & 726,63 \\
\hline & Gravier 4/6,3 & 619,76 & 753,84 \\
\hline & Gravier 6,3/10 & 356,21 & 418,83 \\
\hline & Gravier 10/14 & 141,34 & 167,73 \\
\hline & Adjuvant & - & $0,25 \%$ \\
\hline \multicolumn{2}{|r|}{ Rapport E/C } & 0,6 & 0,35 \\
\hline
\end{tabular}

Les éprouvettes cylindriques (11X22) destinées aux essais de capillarité, de porosité à l'eau et de masse volumique, sont préalablement sciées pour obtenir des disques de la partie médiane d'une épaisseur de $5 \mathrm{~cm}$. Elles sont ensuite séchées à l'étuve à une température de $100^{\circ} \mathrm{C}$ jusqu'à stabilisation de la masse (variation de masse entre deux pesées espacées de $24 \mathrm{~h}$ inférieure à $0,1 \%$ ) puis ramenées à la température ambiante dans un dessiccateur.

Les échéances d'essai pour la durabilité sont 28 jours et $1 \mathrm{an}$, pour les mesures de résistance mécanique elles sont 28jours, 6 mois et 1 an.

Tableau 4 : Nomenclature des bétons

\begin{tabular}{|l|l|}
\hline Bétons & Dénomination \\
\hline Béton ordinaire témoin & BOT \\
\hline $\begin{array}{l}\text { Béton ordinaire avec du laitier } \\
\text { mais sans fibres }\end{array}$ & BOL \\
\hline $\begin{array}{l}\text { Béton ordinaire avec du laitier et } \\
\text { des fibres avec 0,3\% d'adjuvant }\end{array}$ & BOF \\
\hline Béton de hautes performances témoin & BHPT \\
\hline $\begin{array}{l}\text { Béton de hautes performances avec } \\
\text { du laitier mais sans fibres }\end{array}$ & BHPL \\
\hline $\begin{array}{l}\text { Béton de hautes performances avec } \\
\text { du laitier et des fibres avec 0,5\% } \\
\text { d'adjuvant }\end{array}$ & BHPF \\
\hline
\end{tabular}

\section{Résultats et discussions}

\subsection{Effet des réactions sulfatique internes sur les résistances mécaniques}

La diminution des résistances mécaniques est la conséquence des réactions chimiques et des modifications microstructurales causées par l'attaque sulfatique [12]. La Figure 1 exprime l'évolution de la résistance à la traction par flexion des bétons élaborés en fonction de leur composition et de leur âge. De la même manière, la figure 2 présente la résistance à la compression. Ces figures permettent d'estimer l'effet de l'attaque sulfatique interne sur les performances mécaniques des bétons d'étude.

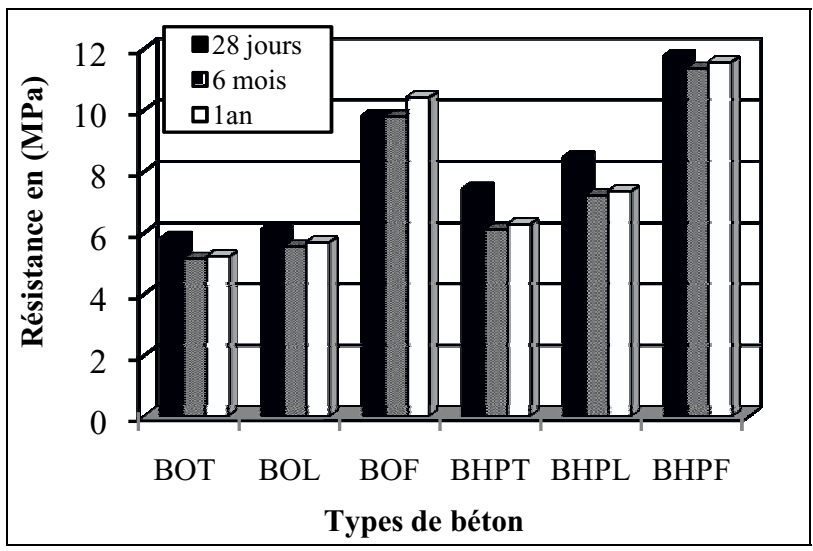

Fig.1 : Résistances à la traction par flexion des différents bétons

A partir des résultats obtenus, on peut constater que les bétons avec ajouts de laitier présentent des résistances plus importantes que les bétons témoins. Pour les résultats de l'essai de traction par flexion (figure 1), la chute des résistances entre 28 jours et 6 mois est faible ou négligeable pour tous les bétons, de la même manière on constate une augmentation des résistances entre 6 et 12 mois. Par contre, dans le cas de la figure 2, on observe une légère augmentation de la résistance à la compression entre 28 jours et 6 mois, même entre 6 et 12 mois.

La présence des fibres réduit la résistance à la compression, mais entraîne un accroissement de la résistance à la traction par flexion.

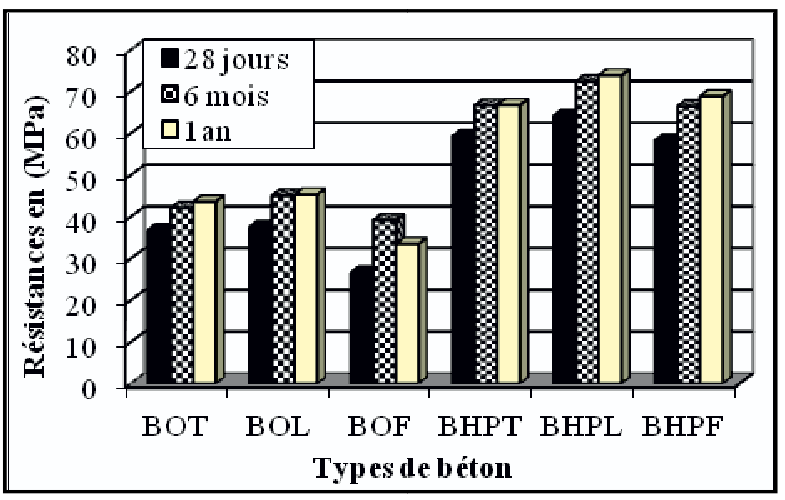

Fig. 2 : Résistances à la compression des différents bétons

\subsection{Effet des réactions sulfatique internes sur les propriétés de transfert (physiques)}

\subsubsection{Absorption d'eau par capillarité}

L'essai d'absorption d'eau par capillarité consiste à placer la face inférieure de l'échantillon au contact de l'eau et de suivre en fonction du temps, l'évolution de la masse. Les figures 3 et 4 illustrent l'évolution de l'absorption d'eau par capillarité des bétons d'étude en fonction du temps. 


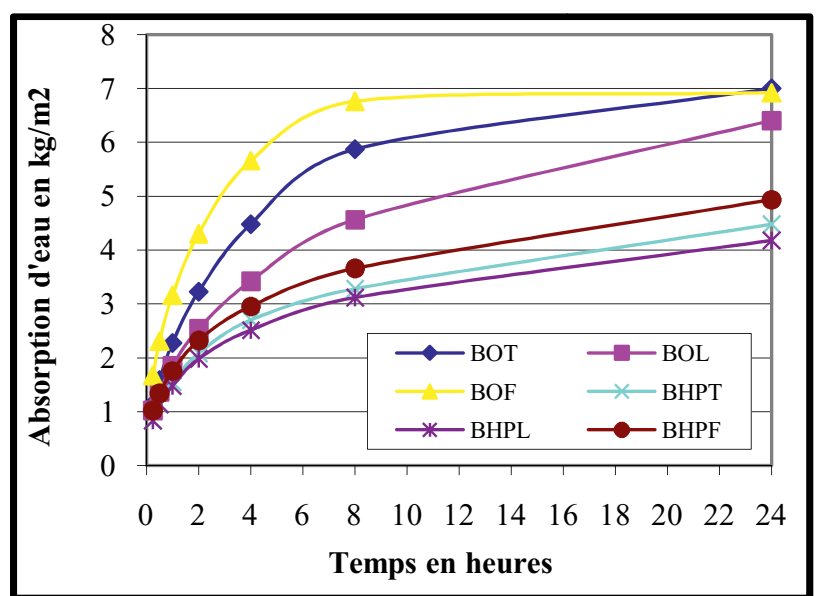

Fig. 3 : Coefficient d'absorption capillaire des différents bétons à 28 jours

Les résultats obtenus montrent que le coefficient d'absorption capillaire des bétons avec ajout du laitier est plus faible que celui des bétons témoins. Les bétons fibrés présentent une absorption d'eau légèrement plus importante. Les coefficients d'absorption capillaire à l'âge d'un an des bétons de hautes performances sont nettement inférieurs comparés à ceux à 28 jours. L'évolution de l'hydratation des bétons peut expliquer ce phénomène.

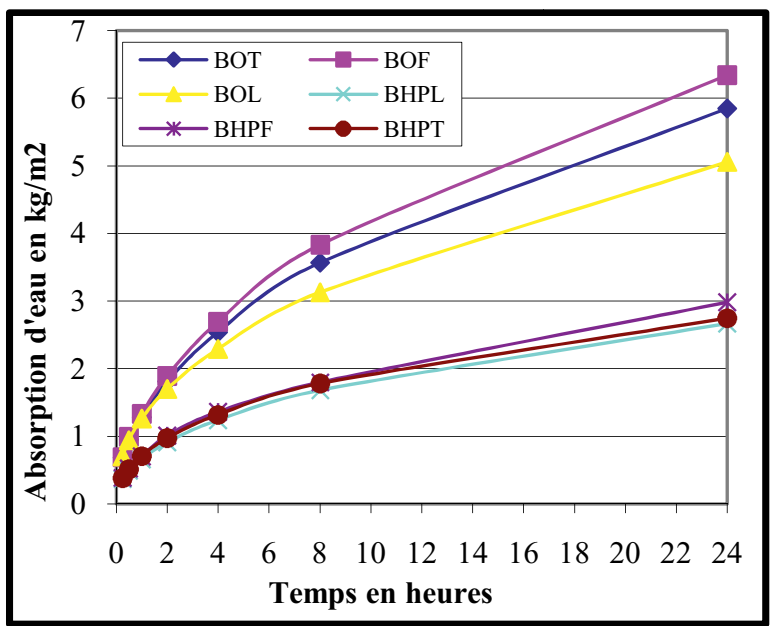

Fig. 4 : Coefficient d'absorption capillaire des différents bétons à 1 an

\subsubsection{Porosité accessible à l'eau et masse volumique}

La porosité ouverte permet de caractériser aisément la durabilité des bétons [13]. Le protocole d'essai utilisé pour la détermination de la masse volumique et de la porosité accessible à l'eau est conforme aux recommandations du groupe AFREM [14]. Les résultats de ces essais sont représentés sur les figures 5 et 6 .

On note une très faible augmentation de la porosité des bétons ordinaires entre l'âge de 28jours et 1 an. Par contre, on observe une légère diminution de la porosité des bétons de haute performance entre 28 jours et 1 an. La porosité des bétons non fibrés est moins importante que celles des bétons fibrés. Il est couramment admis que l'incorporation des fibres dans un BHP conduit à une baisse de compacité liée à la réduction des propriétés rhéologique [15].

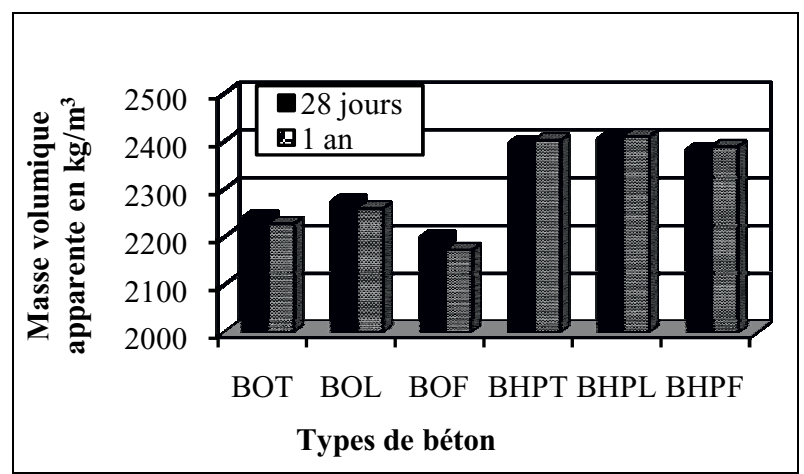

Fig. 5 : Variation de la masse volumique des différents bétons.

Indifféremment du type de béton, la masse volumique augmente avec la diminution de la porosité accessible à l'eau ; cela est vérifié pour les deux types de bétons.

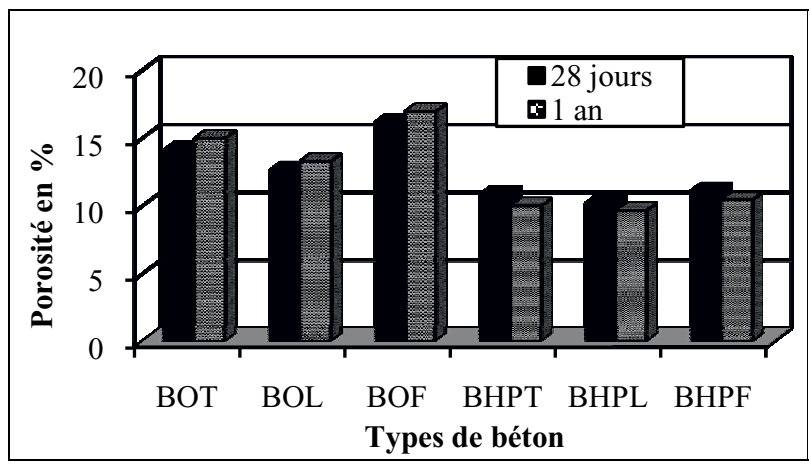

Fig.6 : Variation de la porosité accessible à l'eau des différents bétons

\subsection{Microstructure des éprouvettes de béton examinées au MEB}

La figure 7 présente les micrographies de la microstructure interne des bétons après $1 \mathrm{an}$ de conservation dans la salle humide. Les prélèvements correspondent à des éléments très proches de la surface en contact avec la vapeur d'eau.

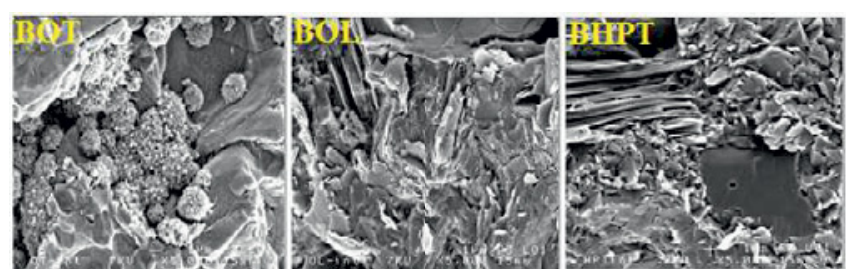

Fig.7 : Microstructure interne des bétons ordinaires et du BHP après lan de conservation dans une salle humide

La figure 7 montre pour le béton témoin ordinaire (BOT) la présence de sulfure sous forme framboïdale qui serait à l'origine de la formation ultérieure d'ettringite [16]. Dans le cas du béton ordinaire au laitier (BOL) et le béton de haute performance témoin (BHPT), la présence 
de sulfure n'a pas été détectée. On retrouve une microstructure classique constituée de $\mathrm{CSH}$ et de portlandite.

Les micrographies de la microstructure externe des bétons ordinaires et du BHP après 1 an de conservation dans la salle humide sont présentées sur la figure 10. Sur les surfaces en contact direct avec l'humidité, on note la présence d'ettringite primaire non expansive pour les trois bétons. Elle est en aiguilles fines pour le béton ordinaire au laitier et en très faible quantité dans le BHP.

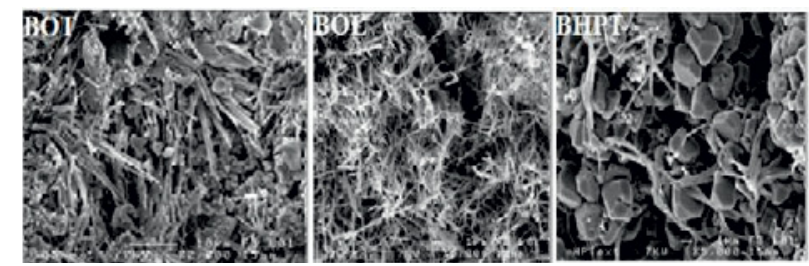

Fig.8 : Observation au MEB de la microstructure externe des bétons ordinaires et du BHP après 1 an de conservation dans la salle humide

\section{Conclusions}

Les résultats des essais montrent une amélioration des caractéristiques physiques et mécaniques des bétons de haute performance fibrés entre les échéances de 28jours et une année. De même, Les micrographies de la microstructure interne et externe de la matrice cimentaire ne montre ni expansion, ni altération des hydrates.

Cette étude montre que le soufre présent dans les granulats de concassage de roches cornéennes n'a entrainé aucune réaction expansive des bétons de haute performance dans nos conditions expérimentales.

Dans les bétons ordinaires, les granulats pyriteux provoquent de la fissuration par expansion lorsqu'ils sont en milieu humide. Dans les bétons à hautes performances ce phénomène est fortement réduit ou inexistant. Ceci est la conséquence d'une porosité très réduite, une circulation de l'humidité réduite qui ne peut se propager jusqu'au granulat. Ainsi le sulfure est stable et ne peut se transformer en sulfate agressif.

\section{References}

1. J.BERARD, R. ROUX, M. DURAND, Performance of concrete containing a variety of black shale. Can. J. Civ. Eng. 2, 58, (1975).

2. R.E.OBERHOLSTER, P.DUTOIT, JL. PRETORIUM, Deteriorationof concrete containing a carbonaceous sulphide-bearing aggregate, National building research institute, CSIR, Pretoria, South Africa, (1984).

3. B.GODART, L. DIVET, Réaction sulfatique interne dans les structures en béton - Mécanisme, pathologie et prévention, Techniques de l'Ingénieur, c2254
4. M.AL SHAMAA, Etude du risque de développement d'une réaction sulfatique interne et de ses conséquences dans les bétons de structure des ouvrages nucléaires, thèse de doctorat, Université Paris-Est, (2012).

5. D.HEINZ, U. LUDWIG, Mechanism of secondary ettringite formation in mortars and concretes subjected to heat treatment, Concrete durability, American Concrete Institute, K \& B Malter, vol. ACI SP-100, Detroit, USA, pp. 2059-2071, (1987).

6. L.GRAF, V.JOHANSEN, Effect of relative humidity on expansion and microstructure of heat-cured mortars, $12^{\text {th }}$ International Congress on the Chemistry of Cement, Montréal-Canada (2007)

7. P-C.AÏTCIN, Bétons hautes performances, édition Eyrolles, (2001).

8. G.ESCADEILLAS, Les éco-matériaux dans la construction : enjeux et perspectives, septième édition des Journées scientifiques du Regroupement francophone pour la recherche et la formation sur le béton (RF) $2 B$, (2006).

9. F-M.MILLER, T. CONWAY, Use of ground granulated blast furnace slag for reduction of expansion due to delayed ettringite formation, Cement Concrete and Aggregates, vol. 25, n $^{\circ}$ 2, pp. 59-68, (2003).

10. T.RAMLOCHAN, P.ZACARIAS, M-D-A. THOMAS, R-D. HOOTON, The effect of pozzolans and slag on the expansion of mortars cured at elevated temperature, Part I: Expansive behavior, cement and Concrete Research, vol. 33, n6, pp. 807-814, (2003).

11. L.DIVET, P.FASSEU, A. SANTOS SILVA,A Optimisation of the choice of cement in order to reduce the expansion of concrete as a result of delayed ettringite formation. $7^{\text {th }}$ CANMET/ACI International Conference on Durability of Concrete, Montreal, Canada, Supplementary papers, pp. 331-342, (2006).

12. M.R.KHELIFA, Effet de l'attaque sulfatique externe sur la durabilité des bétons autoplacants, thèse de doctorat, (2009).

13. A.BESSA, Etude de la contribution des additions minérales aux propriétés physiques, mécaniques et de durabilité des mortiers, thèse de doctorat, Université de Cergy-Pontoise, (2004).

14. G.ARLIGUIE, H. HORMAIN, GranDuBé, Recommandation AFREM «Bétons. Détermination de la masse volumique apparente et de la porosité accessible à l'eau », Presses de l'école nationale des ponts et chaussées, (2007).

15. W.LADAOUI, Etude expérimentale du comportement à long terme des BHP destinés aux ouvrages de stockage des déchets radioactifs, Thèse de doctorats, Université de Toulouse III-Paul Sabatier, (2010). 